\title{
Trabajo infantil e inasistencia escolar
}

\author{
Antonio Sandoval Ávila \\ Universidad de Guadalajara, México \\ Centro de Estudios sobre el Cambio y las Instituciones
}

\section{Introducción}

El trabajo infantil es un fenómeno complejo y multidimensional del que es difícil separar sus componentes sociales, culturales y económicos, pues en cada país o región está estrechamente vinculado con el entorno social, cultural y económico. Sus causas son muy diversas y comprenden tanto factores estructurales como culturales. Las causas estructurales del trabajo infantil actúan en el nivel de la economía y de la sociedad en un sentido amplio al igual que sobre determinadas situaciones, actitudes y valores que pueden predisponer a las familias y/o comunidades a aceptar e incluso fomentar el trabajo infantil. En algunos contextos, especialmente los pobres, el

* El presente trabajo es un producto secundario de un proyecto de investigación terminado intitulado: “Algunos factores que favorecen la expulsión de los hijos del seno del hogar a la calle", que se encuentra actualmente en fase de revisión antes de entregarlo a la editorial de la Universidad de Guadalajara. trabajo es percibido por muchos padres y madres como una suerte de escuela para la vida, como una forma de capacitar a sus hijos no sólo en términos de alguna habilidad o conocimiento, sino para enseñarles lo que es la vida (Alarcón Glasinovich, 2002).

Las razones que explican por qué los niños y niñas trabajan en lugar de estudiar son muy diversas y comprenden tanto los factores estructurales como culturales, desde las vinculadas con la situación socioeconómica de los hogares como la pobreza, pasando por la permanencia de costumbres y normas culturales que no ven la educación de los niños y niñas como una inversión, hasta la falta de infraestructura educativa. En México, entre estas causas se destacan, en orden de importancia, la falta de recursos monetarios, la cual representa $21,1 \%$, luego el trabajo que tienen que desempeñar los niños y niñas para apoyar la economía familiar $10,7 \%$, y los quehaceres domésticos 10,2\%. Las tareas domésticas que realizan los menores permiten que otros miembros del hogar puedan realizar un trabajo remunerado. En conjunto, las razones económicas indican que 42 de cada 100 niños y niñas de 6 a 14 años que trabajan, no estudian 
porque el hogar no dispone de los recursos económicos suficientes para sufragar los costos que su educación implica (INEGI, 2004).

Los efectos económicos y sociales del trabajo infantil también son diversos e inciden tanto en el nivel microfamiliar como en el macroeconómico y social. A nivel microfamiliar, el trabajo infantil incrementa en el corto plazo el ingreso del hogar, pero en el largo plazo disminuye la formación de capital humano, ya que muchos niños y niñas que trabajan no asisten a la escuela, otros la abandonan antes de concluir la educación básica y unos más no continuarán estudiando más allá de este nivel. Esto incide de manera negativa en el nivel educativo de la población y en la productividad de la fuerza laboral y su competitividad. El hecho de que los niños y niñas trabajen reduce sus oportunidades de salir de la pobreza. Al llegar estos niños y niñas a la edad adulta con un nivel de escolaridad bajo, sus oportunidades de empleo se restringen a trabajos poco calificados y de bajos salarios, contribuyendo de esta manera a reproducir los esquemas de organización familiar y las condiciones de pobreza (idem).

El trabajo infantil no sólo es ilegal, moralmente inaceptable y un ultraje a la dignidad humana. No sólo lastima a los niños y niñas que por derecho deberían estar estudiando en lugar de trabajar, sino que además resulta poco rentable en términos económicos. En México, el $65 \%$ de los niños y niñas que trabajan no reciben ningún pago por su par-ticipación en la producción de bienes o la prestación de servicios. Sólo un $6 \%$ reciben un salario mínimo o más. El resto recibe menos de un salario mínimo. Las labores que realizan obedecen en alguna medida a factores económicos y socioculturales vinculados a las estrategias económicas de las unidades familiares, sobre todo en las zonas rurales. De hecho retrasa el desarrollo de los recursos humanos, reduce la duración de la vida activa de los individuos y disminuye el nivel de productividad y crecimiento económico de la sociedad (idem). Por ello la intención de resaltar el impacto que el trabajo infantil tiene sobre la educación de los menores.

\section{El modelo de desarrollo capitalista neoliberal}

El capitalismo neoliberal, que maneja la economía transnacional, desempeña un papel clave en la configuración no sólo de la economía mundial, sino también de la sociedad global en su conjunto. De manera "suave y normal", ha tomado los centros materiales vitales de las sociedades modernas. Pretende, con miras a la realización de la utopía del anarquismo mercantil, servirse de los Estados nacionales para conseguir "pactos" con miras a lograr condiciones impositivas más suaves y unas infraestructuras más favorables para sus empresas, desmantelando el aparato y las tareas estatales, eliminando las trabas a la inversión, las trabas de los sindicatos, de la normatividad ecológica, asistencial y fiscal. Castiga a los países careros o poco amigos de sus inversiones, ya que por su poder económico las empresas transnacionales pueden invertir en un país, producir en otro, o pagar impuestos en un tercero según les resulte más ventajoso por las altas tasas de interés, o más atractivo por la mano de obra barata, o menos oneroso para pagar sus impuestos.

Este capitalismo, que determina la relación social en todo el planeta, se estructura en buena medida en torno a una red de flujos financieros que dictan el destino de las grandes empresas, las economías regionales, las divisas nacionales; los ahorros familiares, los puestos de trabajo, los salarios, los impuestos y los servicios públicos, socavando así, de manera legal pero ilegítima, el bien general que tanto proclama (Beck, 1998).

Aunque capital y trabajo viven el uno por el otro, no se relacionan entre sí. El capital global depende cada vez más del trabajo genérico prescindible y cada vez menos del trabajo específico. El capital es global. El trabajo es local. Las relaciones de producción quedan desconectadas de su existencia real, y se reintegra su resultado mediante una multiplicidad de tareas interconectadas en emplazamientos diferentes, en una nueva división del trabajo basada en las capacidades de cada trabajador más que en la organización de las tareas (Castells, 1999, v. I). 
Así, el trabajo se individualiza cada vez más, pierde su identidad colectiva, se fragmenta su organización. Ésta se disuelve en una variación infinita de existencias particulares que individualiza las condiciones laborales, los intereses, los proyectos. Con esta individualización del trabajo que socava su organización colectiva, los sectores más débiles de la mano de obra quedan abandonados a su suerte. De esta manera, la globalización de la economía y la desaparición gradual del Estado de Bienestar, bajo el impacto de la individualización del trabajo, priva de una red de seguridad a la gente que no puede alcanzarla de forma individual (idem, v. III).

\section{Consecuencias del modelo de desarrollo capitalista neoliberal}

El deterioro del mercado de trabajo en México y en los demás países de América Latina ha sido la consecuencia más desastrosa de este modelo con el que se ha acentuado el desempleo que golpea especialmente a los jóvenes, y como consecuencia de ello se ha masificado la pobreza (Prats, 2004). En 2003, el Panorama Laboral de la Organización Internacional del Trabajo (OIT) muestra que América Latina, pese a la finalización del ciclo recesivo del 2002 y al asomo de una modesta recuperación económica en el 2003, continúa registrando altos niveles de desocupación, deterioro de la calidad del empleo, aumento de la informalidad en los nuevos puestos de trabajo, caída de los salarios reales y reducción de la productividad de su fuerza laboral (OIT, 2003a).

Según el Banco Interamericano de Desarrollo (BID), en el 2003 el desempleo abierto promedio en la región subió al 10,9\% y en muchos países del área superó el 20\% (Radionoticias, 2004). Para ese año el desempleo en América Latina alcanza a más del 50\% de su población económicamente activa (PEA) (García Morales, 2003). Según el informe anual de la OIT, América Latina cerró el año 2003 con 19 millones de desempleados. Los niveles que alcanza el trabajo informal son muy preocupantes. Uno de cada tres trabajadores latinoamericanos se desempeña en el sector informal (Mora, 2004).
En México, según la Encuesta Nacional de Empleo correspondiente al primer trimestre de 2004, la tasa de desempleo fue de 3,86\% de la PEA (Pescador, 2004). Además, 21 millones de personas, más de la mitad de la PEA, percibe ingresos menores a dos salarios mínimos (CONAPO, 2003). Según la OIT, en México existen 25,5 millones de personas empleadas en la economía informal, de los cuales 17 millones son hombres (67\%) y 8,5 millones son mujeres (33\%) (González Romero, 2005).

El poder adquisitivo del salario mínimo en la región latinoamericana se contrajo. Al menos cuatro de cada diez latinoamericanos perciben ingresos insuficientes para satisfacer sus necesidades básicas (OIT, 2003a). En México, para el año 2002 la pérdida del poder real del salario mínimo era de 85\% (Sotelo Valencia, 2003). Aunque América Latina registró un crecimiento económico de 1,5\% durante 2003, la pobreza alcanzó al $44 \%$ de su población, lo que equivale a 227 millones de latinoamericanos pobres, de los cuales 100 millones se encontraban en la indigencia (Tribin Piedrahita, 2003).

Las cifras estimadas por la Comisión Económica para América Latina (CEPAL) sitúan en el grupo de los países más pobres de América Latina a Honduras con el 79,1\% de su población en pobreza, a Nicaragua con el $67,4 \%$, Paraguay con $61,8 \%$, Bolivia con $61,2 \%$, Guatemala con el $60,4 \%$, Colombia con el $54,9 \%$ y El Salvador con el 49,9\%. En el grupo de países con índices altos de pobreza están Perú con el 49\%, Venezuela con el 48,5\% y México con el 43,3\%. En medio, entre el grupo de países más pobres y el grupo de países con altos índices de pobreza, están Brasil con el 36,9\%, Panamá con el 30,8\% y República Dominicana con el 29,2\% (Pinzón, 2002). ${ }^{1}$

${ }^{1}$ Los métodos de medición de la pobreza se vinculan con la conceptualización que se haga de ella. Estas metodologías no son neutras, contienen elementos subjetivos y en ocasiones la definición de la línea de la pobreza atiende a criterios políticos. Aunque no hay un indicador que sintetice todas la dimensiones de la pobreza, 
El desempleo y la generalización de la pobreza han generado un incremento de la participación económica de las mujeres y de los hijos para afrontar la crisis económica. Las familias pobres tienen condiciones de vida tan difíciles, que éstas hacen imprescindible el trabajo infantil para que las familias puedan subsistir (OIT, 2003b). La tasa de participación femenina aumentó, mientras que la masculina se estancó. En 1998 las mujeres representaban el $40 \%$ de la PEA urbana en la región. La mayor participación femenina se produjo en los grupos de 25 a 44 años (OIT, 2001).

En México, en el año 2000, la tasa de participación laboral masculina era de $76,8 \%$ y la femenina de 36,4\%. En el año 2003 la tasa de participación masculina se redujo a $74,6 \%$ y la femenina al $35,3 \%$ (Damián González, 2004). 35 de cada 100 mujeres participaban en la actividad económica, las comprendidas entre los 25 y 44 años reportan las tasas más altas de participación. La participación de las mujeres casadas o unidas es la que más ha aumentado, su participación actual es de aproximadamente $32 \%$. La mayor participación la tienen las divorciadas, 71 de cada 100 trabajan; les sigue el grupo de separadas con $63,4 \%$, y luego las solteras con $38,3 \%$ (INEGI, 2003). Sin embargo, el incremento del empleo de las mujeres durante la década de los noventa del siglo pasado, $4 \%$ anual, superior al de los hombres, 2,6\%, no fue suficiente para absorber la creciente oferta de mano de obra femenina. La tasa de desempleo femenino fue un $47 \%$ superior al desempleo masculino (OIT, 2001).

\section{Niños que trabajan}

Se calcula que en el mundo más de 260 millones de niños y niñas entre 5 y 17 años de edad trabajan, y que de ellos 128 millones lo hacen en los países en

uno de los métodos más difundidos es el de la medición del ingreso. De acuerdo con este indicador, la OIT fija la línea de la pobreza en un ingreso diario de US\$2 per capita, y la pobreza extrema en US\$1. Este mismo criterio aplica la CEPAL en América Latina y el Caribe (CEPAL, 2004). desarrollo (Ángel, 2002). Estimados de la OIT indican que en América Latina y el Caribe el total de niños y niñas que trabajan es de alrededor de 20 millones, lo que significa que uno de cada 5 menores en la región trabaja. Esta cifra equivale a cerca de una sexta parte de los niños latinoamericanos y representa el 5\% de la PEA de la región (CEDE, 2002).

En México, en el año 2002 el número de niños y niñas de 6 a 14 años de edad que trabajan es de 3,3 millones, que se distribuyen casi por igual entre niños y niñas. De este total, 1,5 millones $(45,3 \%)$ realizan algún trabajo económico y 1,8 millones $(54,7 \%)$ realizan trabajo doméstico. Lo anterior significa que uno de cada seis menores en este rango de edad, desempeña un trabajo, ya sea éste económico o doméstico (INEGI, 2004).

Asumimos las dos definiciones de trabajo infantil que presenta la OIT: una, restringida, que comprende exclusivamente las actividades económicas, y otra, ampliada, que incluye tanto a las actividades económicas como al trabajo doméstico excluyente, es decir, el trabajo doméstico que realizan los menores que, por el número de horas que dedican a esta actividad, interfiere con su asistencia a la escuela o con la atención satisfactoria de las actividades escolares. La OIT señala que el número de horas que incide sobre el aprovechamiento escolar de los menores oscila entre 2 o 3 horas diarias, o sea, unas 15 horas a la semana (idem, 2004).

Por clase de trabajo son notables las diferencias entre niños y niñas. En México en el año 2002, siete de cada diez niños y tres de cada diez niñas entre los 6 y 14 años de edad, desarrollan actividades económicas. En el caso del trabajo doméstico la situación es opuesta, siete de cada diez niñas y tres de cada diez niños realizan trabajos domésticos. La división del trabajo entre niños y niñas en los hogares, no hace más que evidenciar la forma en que se reproducen las pautas sociales y culturales que asignan un papel diferenciado a los hombres y a las mujeres desde temprana edad (idem).

Una tercera parte de los niños y niñas que trabajan tienen entre 6 y 11 años de edad y dos terceras partes 
tienen entre 12 y 14 años. Por grupos de edad se observa que los niños y niñas de 12 a 14 años son más (65,3\% del total de la población de 6 a 14 años) que los niños y niñas de 6 a 11 años (34,7\%). Esta situación muestra que a medida que la edad de la población infantil aumenta, la probabilidad de insertarse en el trabajo económico y doméstico también tiende a aumentar. En el grupo de 6 a 11 años se observa una proporción mayor de niños $(37,2 \%)$ que de niñas $(27,9 \%)$. En el grupo de 12 a 14 años la participación de las niñas es mayor $(72,1 \%)$ que la de los niños (62,8\%). Esto es, de 6 a 11 años son más los niños que trabajan que las niñas, pero de 12 a 14 años la situación es inversa.

El mayor peso que los niños y niñas de 12 a 14 años tienen respecto al total de la población infantil de 6 a 14 años que trabaja, se debe a que muchos de ellos concluyen su educación primaria alrededor de los doce años y no continúan estudiando; ingresan a las actividades económicas o ayudan en las tareas domésticas, y son las niñas las que principalmente son incorporadas desde temprana edad a los quehaceres del hogar (idem). 55\% de los inscritos en primaria la terminan. El resto no continúa estudiando y el $69 \%$ de ellos aduce como razón para ello la falta de recursos económicos que los obliga trabajar (Ruíz Del Castillo, 2002).

De acuerdo con lo dicho arriba, respecto a que al aumentar la edad de los menores la probabilidad de insertarse en el trabajo económico y doméstico también tiende a aumentar, en un estudio sobre el proceso de expulsión de los menores del seno familiar a la calle, en una muestra de 82 familias con niños en la calle de la Zona Metropolitana de Guadalajara, encontramos que, efectivamente, al aumentar la edad de los menores se van incorporando cada vez más al trabajo económico o doméstico y, paralelamente, empiezan también a desertar de la escuela. De un total de 57 menores trabajadores en estas familias, el $25 \%$ tienen entre 12 y 14 años y el 55\% entre 15 y 17 . El $48 \%$ del total de menores entre los 12 y los 17 años de edad trabajan y no asisten a la escuela (Ver Cuadro 1).
Cuadro 1

Asistencia escolar y trabajo en

menores de 6 a 17 años de edad

\begin{tabular}{|l|c|c|c|c|}
\hline Edad & $\begin{array}{c}\text { Sólo asisten } \\
\text { a la escuela }\end{array}$ & $\begin{array}{c}\text { Trabajan y } \\
\text { asisten a } \\
\text { la escuela }\end{array}$ & $\begin{array}{c}\text { Trabajan y } \\
\text { no asisten } \\
\text { a la escuela }\end{array}$ & $\begin{array}{c}\text { No trabajan } \\
\text { no asisten a } \\
\text { la escuela }\end{array}$ \\
\hline 6 a 8 & $88.6 \%$ & - & - & $11.3 \%$ \\
9 a 11 & $67.5 \%$ & $16 \%$ & $4 \%$ & $12.5 \%$ \\
12 a 14 & $63 \%$ & $17 \%$ & $8 \%$ & $11.5 \%$ \\
15 a 17 & $24 \%$ & $15.5 \%$ & $40 \%$ & $20.5 \%$ \\
\hline
\end{tabular}

Fuente: Elaboración propia a partir de datos obtenidos mediante una encuesta a 82 familias con niños en la calle de la Zona Metropolitana de Guadalajara, 2001.

Como puede observarse en el cuadro, no encontramos menores de 6 a 8 años trabajando. La mayoría de los menores en este rango de edad $(88,6 \%)$ sólo asiste a la escuela, el restante $11,4 \%$ no trabaja y no asiste a la escuela. Entre los 9 y los 11 años, estos menores empiezan a incorporarse al trabajo y en un número pequeño empiezan también a desertar de la escuela. Entre los 12 y los 14 años se incorporan más al trabajo y también desertan más de la escuela. Entre los 15 y los 17 años el porcentaje que asiste a la escuela y no trabaja se reduce a un $24 \%$, mientras que los que trabajan y no asisten a la escuela se eleva a $40 \%$ y los que no asisten a la escuela ni trabajan se eleva a 20,5\%.

Llama la atención los porcentajes de niños de 6 a 8 años que, debiendo ir a la escuela, no asisten a ella. Esto puede estar en relación con la imposibilidad de los padres para enviar a los hijos a la escuela, por su precaria situación económica. La incorporación al trabajo y la deserción escolar en los menores de 12 a 14 años puede estar en relación con el hecho de que entre los 12 y los 14 años los niños concluyen su educación primaria y muchos de ellos no continúan estudiando, ingresan a las actividades económicas o ayudan, principalmente las niñas, en las tareas domésticas, lo cual permite que otros miembros del hogar puedan incorporarse al trabajo remunerado.

También llama la atención el hecho de que una quinta parte de los menores de 15 a 17 años de edad no trabajan y no asisten a la escuela. Esto podría estar en relación con lo anterior o con el desempleo imperante, que es más acentuado en hombres y 
mujeres jóvenes. Esto resulta preocupante por la gran cantidad de tiempo libre de que disponen estos menores que viven en un medio ambiente precario, carente de centros de recreación, que tienen necesidades económicas y afectivas insatisfechas y que pueden ser enganchados con facilidad en actividades delictivas.

\section{Trabajo infantil y asistencia escolar}

El deterioro del panorama social en América Latina también se evidencia en el plano educacional. Los niños y niñas de la región tienen la posibilidad de ingresar a los sistemas educativos, pero su probabilidad de completar los ciclos escolares está condicionada por su situación socioeconómica. Las deterioradas condiciones de vida y la debilidad del núcleo familiar atentan contra la asistencia a la escuela de los menores y el rendimiento en ella (Kliksberg, 2002). Las dificultades económicas y el trabajo son las principales razones que los jóvenes aducen para el abandono escolar. Entre las mujeres se suman las tareas del hogar, el embarazo y la maternidad (Sánchez, 2002).

Sólo el 14\% de los niños de América Latina asiste a preescolar, instancia educativa considerada crucial por su peso en la formación de estructuras básicas. La tasa de inscripción en la primaria supera el $90 \%$ pero $50 \%$ de los niños que la comienzan desertan de la escuela antes de completar la educación básica. Entre el 25 y el 50\% de los niños que ingresan a primaria no completan el quinto grado, y sólo uno de cada tres que inicia el ciclo secundario lo termina. De acuerdo con la CEPAL, en Brasil en el año 2000 repetían los dos primeros años de la escuela primaria el $45 \%$ de los niños y niñas de la población con menores ingresos y sólo el 4,5\% de los niños y niñas del $25 \%$ con mayores ingresos. Igualmente, a los 20 años de edad habían completado la secundaria el $54 \%$ de los jóvenes del $25 \%$ con mayores ingresos y sólo el $8 \%$ del $25 \%$ de menores ingresos (Kliksberg, 2002).

El nivel de repetición en la región es uno de los más altos del mundo. La tasa de repetición en su con- junto es de 41,2\%. En Brasil es de 45\%, en otros países del cono sur es de 36,3\%. En Centroamérica es de 39,5\%. En República Dominicana, Cuba y México es de 38,6\% (Torres, 2001). Los promedios de repetición llevan a que los niños tarden más de diez años para terminar la primaria en Guatemala, Honduras y Nicaragua. Un alumno promedio en la región está cerca de siete años en la escuela primaria para terminar sólo cuatro grados. Más del $40 \%$ de los alumnos repiten el primer grado, y la tasa de repetición promedio es del $30 \%$ en cada año de estudios (Kliksberg, 2002).

Las elevadas tasas de repetición y deserción llevan a un bajo índice de escolaridad promedio por habitante en la región, el cual es de 5,2 años. Este promedio en algunos países es aún menor, como en Honduras, Paraguay, El Salvador y Brasil, donde el promedio es entre cuatro y cinco años. En los demás países, con sus diferencias, no excede de nueve años. En los países de Centroamérica y en algunos del Caribe, como Haití y República Dominicana, el 83\% de los niños y niñas de los estratos altos terminan el quinto grado de primaria y sólo lo hace el $32 \%$ de los estratos pobres (idem).

A partir de los 12 años comienza a observarse un porcentaje de rezago escolar progresivo conforme aumenta la edad de los menores. A los 12 años se tiene un rezago de $23 \%$, que se eleva hasta el $81 \%$ a los 17 años (OIT, 2003b). Existen, además, fallas en el sistema educativo para retener a la población estudiantil que no logra adaptarse, o que no encuentra en la educación una alternativa compatible con sus intereses y aspiraciones (OIT, 2003c). 42 millones de latinoamericanos son analfabetas (González Romero, 2005).

En América Latina, de acuerdo con el BID, la escolaridad promedio de los jefes de familia del 10\% más rico de la población es de 12 años, mientras la del $10 \%$ más pobre es de 5 años. Hay una brecha de 7 años que tiene efectos en las posibilidades de conseguir trabajo, en los ingresos, etc. En México, esta brecha es de diez años. En Europa, es de dos a cuatro años (Kliksberg, 2002). Alrededor del 20\% de los hijos de las personas con baja escolaridad no logran finali- 
zar la educación secundaria por las condiciones que la pobreza impone a las familias (Prats, 2004).

Según la CEPAL, en el año 2000 la fuerza de trabajo ocupada en la región mostraba una marcada estratificación. Hay un nivel superior que es el 3\% de la población ocupada que tiene 15 años de escolaridad; un nivel intermedio que es el $20 \%$ de la fuerza de trabajo que tiene entre 9 y 12 años de escolaridad; y el 77\% restante tiene sólo de 5 a 7 años de estudios en las ciudades, y de 3 años en las zonas rurales. Diez años de escolaridad parecen constituir el umbral mínimo para que la educación pueda cumplir un papel significativo en la reducción de la pobreza. Si se tiene un nivel inferior y no se poseen activos productivos, son escasas las probabilidades de superar los niveles inferiores de ingreso ocupacional (Kliksberg, 2002).

La diferencia entre los países la hará, de manera creciente, el conocimiento. Un estudio del Banco Mundial (BM) sobre 192 países concluye que sólo el $16 \%$ del crecimiento económico se puede atribuir al capital físico: edificios, maquinaria, infraestructura; el $20 \%$ viene del capital natural y el $64 \%$ puede ser atribuido al capital humano y al social (idem).

En México, más de un millón de niños en edad de cursar la primaria no están inscritos en ella. Del total de la población de 8 a 14 años, el 4,2\% de las mujeres y el 4,8\% de los hombres no saben leer y escribir. En todas las entidades federativas, con excepción de Chiapas, hay más niños que niñas de 8 a 14 años de edad que no saben leer y escribir (INEGI, 2003). En Chiapas se concentra el $27 \%$ de la población entre 6 y 14 años de edad que no asiste a la escuela (Bazdresch Parada, 2001).

En el año 2002, el 12\% de las mujeres y el 9\% de los hombres de 15 años y más carece de instrucción (INEGI, 2003). El 26\% de los analfabetos se concentra en la población indígena (Bazdresch Parada, 2001). A pesar de la disminución del analfabetismo femenino, y de que la brecha entre hombres y mujeres en este sentido es cada vez menor, la diferencia entre sexos aún persiste. La distancia que se observa entre hombres y mujeres que carecen de instrucción formal se debe, al parecer, a la mayor exclusión educativa que sufrieron las mujeres en generaciones pasadas (INEGI, 2003).

A partir de la modificación de los Artículos 3 y 31 fracción 1 de la Constitución Política de los Estados Unidos Mexicanos, en 1993, como respuesta a la necesidad de un mayor nivel de instrucción de la población, la primaria (6 años) y la secundaria (3 años) son obligatorias (durante todo el siglo XX sólo la educación primaria fue obligatoria) y constituyen el nivel de educación básica (idem). En el año 2002 se hizo obligatorio el nivel preescolar ( 3 años). Dos años de preescolar serán obligatorios para el año 2006, y tres para el año 2008 (Fuhman, Javier \& Valencia, 2004).

El 42,8\% de las mujeres y el $42 \%$ de los hombres de 15 años y más tienen educación básica incompleta. El rezago educativo, es decir, el porcentaje de personas de 15 años y más sin instrucción, con primaria incompleta o completa y/o secundaria incompleta, es de $54,3 \%$ para las mujeres y de $50,7 \%$ para los hombres. La brecha entre sexos es, en términos generales, de 3,6 puntos (INEGI, 2003). Llama la atención el alto porcentaje de población joven que no tiene estudios básicos completos, ya que 39 de cada 100 jóvenes de 15 a 29 años se encuentran en situación de rezago educativo y sólo el $25 \%$ del total de la población en estas edades (27,2 millones, que equivale al $28,5 \%$ de la población total del país) asiste a la escuela (idem).

El rezago educativo en 2005 es de 32 millones de personas, de las cuales 6 millones son analfabetos, un millón supera los 45 años, 3 millones son mayores de 65 años, 700 mil son indígenas, y el resto no ha terminado la primaria o la secundaria. Cada año se suman 774 mil jóvenes al rezago educativo (Arana Cervantes, 2005). El analfabetismo y el rezago escolar tienen una relación inversa al tamaño de la localidad de residencia. Conforme es más grande el tamaño de la localidad de residencia el analfabetismo y el rezago escolar tienden a disminuir. En la relación urbano-rural los porcentajes cambian mucho. Los porcentajes urbanos en el área rural se duplican (INEGI, 2003). 
En el año 2000, el promedio de escolaridad nacional para las mujeres es de 7,1 años, y de 7,6 para los hombres. Entre las generaciones más jóvenes, además de que los promedios de escolaridad son mayores, la brecha que hay entre hombres y mujeres disminuye considerablemente. Así, entre la población de 15 a 29 años casi no hay diferencia en el promedio de escolaridad por sexo. El promedio de escolaridad para las mujeres es de 8,6 años, y el de los hombres es de 8,7. A mayor edad, menor es el promedio de escolaridad alcanzado. No tienen secundaria completa en la población de 30 a 44 años el 52,7\% de las mujeres y el $46 \%$ de los hombres. En el grupo de 45 a 59 años, el 74,9\% de las mujeres y el 67,8\% de los hombres. En el grupo de 60 años y más, los porcentajes de población en situación de rezago escolar rebasan al $85 \%$ : $87,7 \%$ para las mujeres y $85,3 \%$ para los hombres. Lo anterior da cuenta de las diferentes oportunidades educativas entre las generaciones. Estas cifras varían con relación a la edad, el área rural y urbana, la etnia y el tamaño de la población (idem).

La reprobación en todos los niveles es mayor en los hombres que en las mujeres, y estos porcentajes aumentan para ambos sexos a medida que se asciende en los niveles educativos. En la primaria los porcentajes de reprobación son: 5,1\% para las mujeres y 7,4\% para los hombres. En secundaria: $14,4 \%$ para las mujeres y 26,2\% para los hombres. En la educación media superior, donde se forma al profesional técnico y al bachiller, se presentan los mayores porcentajes de reprobación. Los porcentajes a escala nacional son, para el profesional técnico: 19,4\% para las mujeres y $28,4 \%$ para los hombres. Los porcentajes en el bachillerato son: $34,3 \%$ para las mujeres y $44 \%$ para los hombres (idem).

La deserción escolar en educación básica y educación media superior, es mayor en los hombres que en las mujeres. La diferencia entre hombres y mujeres que abandonan la escuela se incrementa conforme se avanza en los niveles escolares. Para la primaria los porcentajes de deserción escolar son de 1,7\% para las mujeres y de $2 \%$ para los hombres. En este nivel la diferencia entre hombres y mujeres es de 0,3 puntos porcentuales. En la secundaria los porcentajes son de $6,2 \%$ para las mujeres y de $9,6 \%$ para los hombres. En este nivel la diferencia entre hombres y mujeres es de 3,4 puntos porcentuales. Para el profesional técnico los porcentajes son: $22,1 \%$ para las mujeres y $28 \%$ para los hombres. En este nivel la brecha entre hombres y mujeres es de 5,9 puntos. Los porcentajes para bachillerato son: $13,9 \%$ para las mujeres y 20,2\% para los hombres. Es en este nivel donde se registra la mayor diferencia porcentual entre sexos, con 6,3 puntos. A partir de la secundaria se observan más los casos de abandono escolar. El nivel educativo con mayor deserción es el medio superior. Las causas están vinculadas con las crecientes necesidades económicas de los hogares, pues por lo general son más de origen socioeconómico que escolar (idem).

Para la eficiencia terminal, que se mide por el número de alumnos que terminan un determinado nivel educativo en el tiempo programado para ello, los porcentajes para la primaria son: $87,5 \%$ para las mujeres y $85,5 \%$ para los hombres. Para secundaria: $81,0 \%$ para las mujeres y $71,1 \%$ para los hombres. Para profesional técnico: $47,4 \%$ para las mujeres y 40,1\% para los hombres Para el bachillerato: 63,7\% para las mujeres y $54,3 \%$ para los hombres. Es en las carreras técnicas donde se observan los menores porcentajes de eficiencia terminal, y aquí la diferencia entre hombres y mujeres es de 7,3 puntos porcentuales (idem).

Aún cuando es difícil determinar si los niños y niñas no van a la escuela porque deben trabajar, o trabajan porque no estudian (OIT, 2003b), algunos estudios han demostrado que hay una clara relación entre el trabajo infantil y la exclusión educativa. Durante la educación primaria, los menores trabajadores presentan un retraso de dos a tres grados con relación a los menores que no trabajan (OIT, 2003c).

En México, la incidencia del trabajo infantil sobre la educación de los niños y niñas es significativa: una cuarta parte de los niños y niñas entre los 6 y 14 años de edad que trabajan no estudian, y 78,5\% de ellos destina 15 o más horas a la semana al trabajo, lo cual 
implica que una parte importante de esta población no está en condiciones de cumplir en forma adecuada sus actividades escolares y el trabajo puede llegar al extremo de excluirlos de la escuela y marginarlos posteriormente del mercado laboral, dado que carecen de las competencias necesarias para insertarse en puestos de trabajo con buenos niveles salariales.

De los niños y niñas de 6 a 14 años de edad que trabajan y no estudian, el porcentaje de niños es ligeramente menor (1,3 puntos porcentuales) al que reportan las niñas: $24,9 \%$ de los niños contra $26,2 \%$ de las niñas. Por grupo de edad, el porcentaje de niños y niñas de 6 a 11 años es de 8,7\%; en cambio, en el grupo de 12 a 14 años dicho porcentaje casi se cuadruplica, al llegar a 33,7\%. El comportamiento por sexo muestra que los niños de 6 a 11 años representan el $10,4 \%$, y las niñas el $6,3 \%$, datos que, al compararlos con el grupo de 12 a 14 años, muestran que el porcentaje de niños se triplica, llegando hasta el 33,5\%, y el de las niñas se quintuplica, alcanzando $33,9 \%$ Es decir, en el grupo de 6 a 11 años el porcentaje de niños que trabajan y no estudian es superior al de las niñas, pero en el grupo de 12 a 14 años el porcentaje de niñas que trabajan y no estudian es superior al de los niños. Esto parece evidenciar cierta discriminación de género ante la preferencia de educar más a los niños que a las niñas, situación que se acentúa después de los 12 años (INEGI, 2004).

\section{Conclusiones}

La adopción de las ideas económicas neoliberales en los Estados nacionales de América Latina como la única posibilidad para el desarrollo ha producido resultados desastrosos. En esta situación, culpar al capitalismo neoliberal de los problemas como el de la pobreza, el trabajo infantil y la deserción escolar, puede resultar reconfortante psicológicamente, pero las causas fundamentales de estos problemas son internas y se tienen que resolver internamente. La pobreza que estas ideas han generado no es una maldición inevitable. Es producto de decisiones y políticas humanas. Que no haya pobres, y por tanto trabajo infantil y deserción escolar, depende de cada sociedad, de cómo se organice, de que haga lo necesario para ello.

Las familias pobres, que viven al día, movilizan todos los recursos a mano para sobrevivir, su preocupación está centrada en cómo hacer para comer y subsistir hoy. En estas circunstancias, el trabajo infantil en el corto plazo parece una salida o alivio a la pobreza familiar, porque la ayuda que los niños y niñas aportan al hogar, ya sea a través del ingreso que logran mediante actividades remuneradas, o mediante el trabajo doméstico que desempeñan en el hogar, que permite que otros miembros de la familia puedan desempeñar algún trabajo remunerado, de algún modo contribuye a paliar las carencias de sus familias (Gattino, 1999).

Muchas actividades que realizan los menores son de apoyo a las necesidades de hogares que necesitan contar con mano de obra para el negocio familiar, como ayuda en el trabajo que desempeñan los padres o en las actividades agropecuarias en la agricultura de subsistencia, en labores que, según la opinión de los adultos, no ponen en riesgo su salud e integridad, las cuales además, les permiten adquirir un conjunto de habilidades y destrezas útiles para la vida adulta (Vicherat, 2002).

Desde la situación de las familias empobrecidas y de los pequeños que trabajan, el trabajo infantil tiene una racionalidad que hay que entender. La actitud de los jefes de familia hacia el trabajo de los hijos menores está condicionada por su educación formal; se supone que un mayor nivel educacional de los jefes de familia les ayudaría a privilegiar en los hijos el rol de estudiantes en desmedro del de trabajador. Pero la baja escolaridad de los jefes de las familias pobres alienta la incorporación temprana de los hijos al trabajo. Su bajo nivel de escolaridad no les ayuda para reflexionar acerca del peligro o daño futuro del trabajo infantil y sobre el hecho de que sus hijos dejen la escuela por el trabajo; en todo caso, la necesidad los obliga a recurrir a la fuerza de trabajo de los hijos.

Sin embargo, trabajar implica para los menores un esfuerzo que no sólo consume y quita tiempo y posibilidades de hacer las tareas escolares, también 
les resta energías durante las clases como en la casa para estudiar. El trabajo prematuro les resta oportunidades, traba su asistencia a la escuela, y si ésta no los expulsa por su inasistencia o por su bajo rendimiento, ellos la abandonan por necesidad. Si bien es cierto que en América Latina ha crecido la matrícula educativa, la precaria situación familiar no permite a toda la población infantil pobre acceder a la escuela y/o mantenerse en el sistema educativo. La disminución del ingreso en las familias pobres merma las posibilidades para los niños y niñas para aprovechar los beneficios de la educación. Sin estos beneficios se quedan también sin herramientas para ingresar en el mercado laboral adulto.

En el corto plazo no es fácil percibir el daño que produce el trabajo infantil ni en el ámbito individual ni al nivel del desarrollo de un país en su conjunto. Sin embargo, en el mediano y largo plazo lo que se gana o se pierde en educación dura para toda la vida. El actual niño trabajador, por la pérdida en educación, sólo podrá acceder a las ocupaciones de menor calificación y peor pagadas; por ello, tiene altas probabilidades de ser el futuro padre de nuevos niños trabajadores, reproduciendo así intergeneraciones la pobreza (Del Álamo, 2002).

Educar a nuestros menores puede constituir el primer paso para romper con el círculo vicioso de la pobreza. El papel de la educación es central para reducir la pobreza y promover mayor equidad en el acceso de oportunidades de bienestar, mediante la formación de sujetos con capacidad de insertarse con mejores ingresos en el mundo laboral, de participar activamente en espacios decisorios y de ejercer sus derechos políticos en una democracia participativa.

Si bien no se puede demostrar que la educación "saca" a los pobres de la pobreza, tampoco se puede demostrar que se puede prescindir de ella en el combate a la pobreza. La educación no resuelve todos los problemas, pero sin ella el cambio social para lograr una sociedad más justa será muy difícil. Sin educación, las posibilidades de exigir el cumplimiento de los derechos sociales, económicos, culturales, civiles y políticos son mucho más estrechas; es por ello que la educación abre las puertas a otros derechos. Sin educación no hay ciudadanía posible y sin ciudadanía toda posibilidad de democracia es una quimera.

Aunque la elevación del nivel educativo no se refleja de inmediato en el mejoramiento del nivel de vida, en el mediano y largo plazo el mejoramiento de la educación tiene su influencia. Sólo por mencionar un ejemplo, con la educación de las mujeres disminuye el número de hijos por mujer, el número de embarazos en adolescentes, el número de embarazos no deseados y el número de abortos provocados; mejora la atención obstétrica y disminuye la mortalidad materna; mejora la atención y el cuidado de los recién nacidos, la esperanza de vida de los menores, y disminuye la mortalidad infantil; mejora la alimentación de la familia, el desempeño de los menores en la escuela, y contribuye a conformar, en los miembros de la familia, una conciencia medioambiental. ${ }^{2}$

El papel de la educación ha sido el eje fundamental para contribuir al cambio social y económico en el mundo. En países que han logrado un buen desarrollo, como algunos del sudeste asiático (Singapur, Hong Kong etc.), la educación ha sido uno de los factores que más han contribuido para ello. La elevación del nivel educativo de una sociedad tiene

${ }^{2}$ Sobre metas y programas de apoyo para el desarrollo regional y por país; sobre indicadores de educación y de salud, sobre las ventajas y desventajas de apoyar o no suficientemente estos rubros, sobre trabajo infantil, puede consultarse, entre otros: BM: Quality of Growth, Washington, D. C., 2000; BID: Infancia, Informe Especial, Washinton, D. C., 1999, CEPAL: Panorama Social de América Latina y el Caribe, Santiago de Chile, 2005; Organización Mundial de la Salud (OMS): The World Health Report, Ginebra, 2000; Organización Panamericana para la Salud (OPS): Situación de la Salud en las Américas. Indicadores Básicos, Washington, D. C., 2000; Programa de Naciones Unidas para el Desarrollo (PNUD): Superar la pobreza humana, Nueva York, 2000, PNUD: Informe Anual 2002 (los más pobres en materia de salud y educación), Nueva York, 2001; Fondo de Naciones Unidas para la Infancia (UNICEF): The State of de World Children, Nueva York, 2001. 
múltiples efectos económicos. Hay una significación correlativa entre los niveles de educación y las remuneraciones que las personas pueden alcanzar, y entre el nivel económico social y el capital humano. El aumento de conocimientos y habilidades incrementa los niveles de productividad y facilita la adquisición de nuevas tecnologías (Castells, 1999, v. III).

En el actual contexto mundial de creciente globalización y cambio tecnológico, donde la formación se convierte en eje central para que un país pueda competir, el trabajo infantil, por la pérdida que implica en educación, se constituye en un problema con profundas repercusiones macroeconómicas. No hay posibilidad para que un país pueda ser competitivo si los niños y niñas, en lugar de concentrar su tiempo y esfuerzo en la escuela, tienen que trabajar. Con ello se traba el desarrollo nacional. El desarrollo descansa en el acelerado cambio tecnológico. Pero la tecnología no es más que el conocimiento científico aplicado a la producción. Crear conocimiento supone educación superior, y en la base de este andamiaje está la educación básica, que es el cimiento de cualquier modelo de desarrollo que aspire a la equidad.

Por eso, no mejorar la educación de nuestros menores significa desperdiciar la formación de nuestro capital humano. Por ello, el trabajo infantil no es un problema personal o familiar, sino social. El tema social, más que de recursos, es con frecuencia de prioridades. Sociedades con recursos limitados han obtenido excelentes logros en el campo social y en el de la familia.

Junto al crecimiento económico surge la necesidad de lograr el desarrollo social. Desarrollo económico y social deben ir juntos para que haya desarrollo real. El crecimiento económico es fundamental para posibilitar el avance social, pero sin inversión continuada en educación no habrá capital humano calificado como motor básico de la productividad y la competitividad. Sin cultura como parte integrada del desarrollo, los aparentes logros económicos pueden ser efímeros.

Los proyectos educativos tienen que ir de la mano de políticas económicas y sociales que tiendan a la reorganización de la estructura distributiva, a recomponer la desigualdad recuperando el trabajo como motor de los estilos de desarrollo. El mejoramiento de nuestros niños y niñas va de la mano del mejoramiento de sus familias, y éste no puede ser ajeno al desarrollo social. Por ello, el interés en las familias de nuestros niños y niñas que trabajan no es sólo un interés privado, sino que adquiere necesariamente una dimensión colectiva y pública. Es desde las familias que debe afrontarse la situación de colapso del ingreso y de la fragilidad social a que se ha llevado no sólo a nuestras familias, sino a la sociedad en general. Lo anterior implica la reactivación productiva para el crecimiento del empleo y el nivel de los salarios para los adultos.

Desde esta perspectiva, la actual política económica no es la solución a nuestros problemas en tanto no se modifique la distribución del ingreso. No obstante, no se ve una política clara a largo plazo que garantice la reestructuración productiva que siente las bases para un crecimiento sostenible. No se ve un empeño suficientemente adecuado para reducir el rezago educativo a un ritmo apropiado.

\section{Referencias bibliograficas}

ÁNGEL, Gonzalo. Análisis del último informe de la OIT sobre el trabajo infantil en el mundo. Rel-VITA, Montevideo, Uruguay, 2002. Disponível em: <http://www.re-vita/old/international-futuro-siu.htm>. Acesso em: enero 2004.

ALARCÓN GLASINOVICH, Walter. ¿Por qué erradicar el trabajo infantil? Revista Electrónica DHIAL, Barcelona: Instituto Internacional de Gobernabilidad, n. 33, 3 sept. 2002. Disponível em: <http://www.iigov.org/dhial/dh33_01.htm>. Acesso em: 20 mayo 2004.

ARANA CERVANTES, Marcos Dejar de hacer lo mismo para lograr resultados diferentes. Diario El Informador, Guadalajara, Jalisco, México, 19 jun. 2005.

BAZDRESCH PARADA, Miguel. Educación y pobreza: una relación conflictiva. In: ZICCARDI, Alicia (Comp.). Pobreza, desigualdad social y ciudadanía. Los límites de las políticas sociales en América Latina.Argentina: Consejo Latinoamericano de Ciencias Sociales,, 2001. Disponível em: <http://68,96.200.17/ ar/libros/pobreza/parada.pdf $>$. Acesso em: 15 dic. 2003. 
BECK, Ulrico. ¿Qué es la globalización? Barcelona: Paidós, 1998. CASTELLS, Manuel. La era de la información - v. I e v. III. México: Siglo XXI, 1999.

CEDE - Centro de Estudios para el Desarrollo Económico. La realidad del trabajo infantil en Colombia. Bogotá, Colombia: Universidad de los Andes, CEDE, 2002. Disponível em: <http:// www.encolombia.com/larealidad2_pediatria34-1htm>. Acesso em: 20 oct. 2003.

CEPAL - Comisión Económica para América Latina. El avance de la pobreza en América Latina: Chile Hoy, Santiago de Chile: CEPAL, 2002. Disponível em: <http://www.chile-hoy.de/internacional/41102_pobreza_AL.htm>. Acesso em: 18 dic. 2003.

. Entender la pobreza desde la perspectiva de género.

Santiago de Chile: Fondo de Desarrollo de Naciones Unidas para la Mujer(UNIFEM), 2004. Disponível em: <http://www.fidamerica.cl/ getdoc.php?docid=3251>. Acesso em: 10 enero 2005.

CONAPO - Consejo Nacional de Población. La precarización del empleo. México: CONAPO, 2003. Disponível em: <http:// www.conapo-gob.mx/prensa2002mzo07.htm>. Acesso em: 16 ju. 2004.

DAMIÁN GONZÁLEZ, Araceli. ¿No hay crisis en el empleo en México? Diario El Financiero, México, p. 42, 15 marzo 2004.

DEL ÁLAMO, Oscar. Los niños del Perú. Cómo afrontar el trabajo infantil desde la cooperación al desarrollo. Magazine DHIAL, Barcelona: Instituto Internacional de Gobernabilidad, n. 14, p. 13, 2002. Disponível em: <http://www.iigov.org/dhial/dh14/ todo.htm>. Acesso em: 17 nov. 2003.

FUHMAN, Susana; JAVIER, Murillo F; VALENCIA, Sergio Martinic. Revisión nacional de investigación y desarrollo educativo. México: Organisation for Economic Co-operation and Development, 2004. Disponível em: <http://www.oecd.org/dataoecd/ 42/23/32496490.pdf>. Acesso em: 20 enero 2005.

GARCÍA MORALES, Federico. El modelo neoliberal y la pobreza en América Latina. México: Revista electrónica Globalización, oct. 2003. Disponível em: <rcci.net/globalizacion/fg005.htm-6k$>$. Acesso em: 20 enero 2004.

GATTINO, Silvia y Nora Aquino. Las familias de la nueva pobreza. Buenos Aires: Espacio Editorial, 1999.

GONZÁLEZ RUIZ, Salvador Alejandro. Atrapada, la economía mexicana. Gaceta Universitaria, Universidad de Guadalajara, Guadalajara, Jalisco, México, p. 16, 15 mar. 2004.

GONZÁLEZ ROMERO, Víctor Manuel. País de desertores. Diario El Informador, Guadalajara, Jalisco, México, p. 4, 15 mar. 2005.
INEGI - Instituto Nacional de Estadística, Geografía e Informática. Mujeres y hombres en México. México: INEGI, 2003. . El trabajo infantil en México, 1995-2002. México: INEGI, 2004.

KLIKSBERG, Bernardo. Hacia una economía con rostro humano. 2. ed, 1. reimp. Buenos Aires: FCE, 2002.

MORA, José Eduardo. Trabajo-América Latina. Pocos y malos empleos nuevos. Inter Press Service News Agency, p. 1, 2004. Disponível em: <http://www.ipsenespanol.net/ print.asp?idnews=25744> . Acesso em: 24 enero 2005 .

OIT - Organización Internacional del Trabajo. Panorama laboral 1999. Mejora la situación laboral de las mujeres pero aún persisten fuertes desigualdades respecto a los hombres. Lima, Perú: Oficina Regional OIT, 2001. Disponível em: <http/www.oit.org.pespanish/ 260ameri/publ/panorama/1999/temaesp.html>. Acesso em: 27 marzo 2003.

19 millones de trabajadores urbanos están desocupados en América Latina. Lima, Peru: OIT - Oficina Regional para América Latina y el Caribe, 2003a. Disponível em: <http:// www.oit.org.pe/portal/noticias.php?docCodigo=69>. Acesso em: 25 mayo 2004.

Informe nacional de los resultados de la encuesta de trabajo infantil y adolescente en Costa Rica. San José, Costa Rica: Programa Internacional de Erradicación del Trabajo Infantil (IPEC), 2003b. Disponível em: <http://www.ipec.oit.cr/ipec/ region/paises/costarica.shtml>. Acesso em: 24 jul. 2004. Estudio a profundidad del trabajo infantil y adolescente y la educación en Costa Rica. San José, Costa Rica: Programa Internacional de Erradicación del Trabajo Infantil (IPEC), 2003c. Disponível em: <http://www.ipec.oit.or.cr/ipec/region/ paises/costarica.shtml>. Acesso em: 16 agosto 2004.

PESCADOR, Fernando. Desempleados 1.2 millones. Diario Publico, Guadalajara, Jalisco, México, 15 mayo 2004.

PINZÓN, Martha Lucía. Informe CEPAL 2002: se agudiza la pobreza en América Latina. Santiago de Chile: Comisión Económica para América Latina (CEPAL), 2002. Disponível em: $<$ www.americasnet/commentators/martha_pinzon/pinzon_85-spapdf>. Acesso em: 30 mayo 2004.

PRATS, Joan. Ojo al dato: otra mirada sobre la globalización. Revista Internacional de Gobernabilidad para el Desarrollo, Barcelona: Instituto Internacional de Gobernabilidad, n. 0, p. 1, 3, 11 mar. 2004. Disponível em: 〈http://www.iigov.org>. Acesso em: 25 enero 2005. 
RADIONOTICIAS. El desempleo en América Latina en el 2003 de acuerdo con el BID fue de 10.9\%. Lima, Perú: Radionoticias, 2004. Disponível em: <Radio1160.tema.compe/modulos.php? name $=$ news $\&$ file $=$ print $\&$ sid $=21987-4 k>$. Acesso em: 8 enero 2005 . RUÍZ DEL CASTILLO, Amparo. Crisis, educación y poder en México. 4. reimpr. México: Editorial Plaza y Valdés, 2002.

SÁNCHEZ, Alicia América Latina: imparable la pobreza. Revista electrónica Tierramerica, México, 2002. Disponível em: <http:// www.tierramerica.net/2002/1110/noticias4.shtml>. Acesso em: 10 sept. 2003.

SOTELO VALENCIA, Adrián. La reforma laboral en México. Revista electrónica española Rebelión, México, 2003. Disponível em: <www.rebelion.org/economia/030823abascal.htm>. Acesso em: 2 oct. 2004.

TORRES, Carlos Alberto. Grandezas y miserias de la educación latinoamericana del siglo XX. In: (Comp.) Paulo

Freire y la agenda de la educación latinoamericana en el siglo XXI. Buenos Aires: CLACSO, 2001. Disponível em: <http:// www.clacso.org/wwwclacso/espanol/html/biblioteca/fbiblioteca/ html>. Acesso em: 25 agosto 2004.

TRIBIN PIEDRAHITA, Guillermo. La pobreza aumentó en América Latina. 2003. Disponível em: <www.elalmanaque.com/ actualidad/gtribin/art268.htm>. Acesso em: 13 sept. 2004.

VICHERAT, Daniela. Trabajo infantil y escolaridad en América Latina. Revista Electrónica DHIAL, Barcelona: Instituto Internacional de Gobernabilidad, n. 26, 29 enero 2002. Disponível em: <http:// www.iigov.org/dhial/dh26/todo.htm>. Acesso em: 30 nov. 2003.
ANTONIO SANDOVAL ÁVILA, médico cirujano por la Universidad Nacional Autónoma de México (UNAM) con especialidad en ginecología y obstetricia por la misma Universidad y el Instituto Mexicano del Seguro Social (IMSS). Maestro en sociología de la educación por el Instituto Superior de Investigación y Docencia para el Magisterio (ISIDM) de la Secretaría de Educación Pública del Estado de Jalisco. Doctor en ciencias políticas y sociales por el Centro de Investigación y Docencia en $\mathrm{Hu}$ manidades del Estado de Morelos (CIDHEM), en convenio con la Universidad de Guadalajara. Profesor investigador del Centro de Estudios sobre el Cambio y las Instituciones (CESCI) del Departamento de Sociología del Centro Universitario de Ciencias Sociales y Humanidades de la Universidad de Guadalajara. Miembro del Sistema Nacional de Investigadores (SNI) del Sistema Nacional de Ciencia y Tecnología (CONACYT). Publicaciones: Pobreza y niños en la calle (Guadalajara: Universidad de Guadalajara, México, 1999); Propuesta metodológica para sistematizar la práctica profesional del trabajo social (Buenos Aires: Espacio Editorial, 2001); Prácticas de crianza de familias de la Zona Metropolitana de Guadalajara que tienen niños en la calle (Guadalajara: Universidad de Guadalajara, México, 2007). Proyecto de investigación: "Violencia contra la mujer en la relación de pareja". E-mail: antonios@csh.udg.mx

Recebido em novembro de 2005 Aprovado em outubro de 2006 


\section{Resumos/Abstracts/Resumens}

Antonio Sandoval Ávila

\section{Trabajo infantil e inasistencia escolar}

El capitalismo neoliberal ha generalizado la pobreza que obliga a muchas familias a recurrir al trabajo de los hijos para poder subsistir. Por el trabajo, los menores desertan de la escuela. Cuando adultos, por la pérdida en educación, sólo podrán acceder a las ocupaciones de menor calificación y peor pagadas. Por ello, tienen muchas probabilidades de ser los futuros padres de nuevos niños trabajadores reproduciendo intergeneracionalmente la pobreza. La educación es el primer paso para romper el círculo de la pobreza; hay relación entre los niveles de educación y las remuneraciones que las ersonas pueden alcanzar. El desarrollo descansa en el acelerado cambio tecnológico que no es más que el conocimiento científico aplicado a la producción. Crear conocimiento supone educación superior, y en la base de ésta está la educación básica, que es el cimiento de cualquier modelo de desarrollo que aspire a la equidad. No educar a los menores significa desperdiciar la formación de capital humano, ello traba el desarrollo nacional. Sin educación no habrá capital humano calificado como motor básico de la productividad y la competitividad.

Palabras claves: capitalismo neoliberal; pobreza; trabajo infantil; deserción escolar

\section{Child labour and the lack of school support}

Neoliberal capitalism has generalized the poverty that forces many families to resort to the work of their children to guarantee subsistence. Work results in the children abandoning school and as adults, as a consequence of their lack of formal education, they will only be able to accede to the worse paid occupations requiring low qualification and receiving low wages. As a result, they will probably be the parents of new children workers reproducing intergenerational poverty. Education is the first step in breaking the vicious circle of poverty. There is a correlation between the levels of education and those of remuneration which people can attain. Development depends on accelerated technological change which is no more than scientific knowledge applied to production. The creation of knowledge presupposes higher education whose foundation is elementary education which is the platform of any model of development that aspires to equity. Not educating children signifies wasting the formation of human capital which, in its turn, brakes national development. Without education it will be impossible to qualify human capital, understood as the basic motor of productivity and competitiveness. Key words: neoliberal capitalism; child labour; lack of school support; school drop-out

\section{Trabalho infantil e inassistência} escolar O capitalismo neoliberal generalizou a pobreza, obrigando muitas famílias a 
recorrer ao trabalho dos filhos para garantir sua subsistência. Pelo trabalho, as crianças abandonam a escola. Quando adultos, pela perda da educação, só conseguem ocupações que exigem menor qualificação e pelas quais recebem menores ganhos. Em conseqüência, provavelmente serão pais de novas crianças trabalhadoras, reproduzindo intergerações a pobreza. A educação é o primeiro passo para romper o círculo vicioso da pobreza; há relação entre os níveis de educação $e$ os níveis de remuneração que as pessoas podem alcançar. O desenvolvimento baseia-se no acelerado desenvolvimento tecnológico que nada mais é que o conhecimento científico aplicado à produção. Criar conhecimento supõe educação superior, e seu fundamento está na educação básica, que é a plataforma de qualquer modelo de desenvolvimento que aspire à eqüidade. Não educar as crianças significa desperdiçar a formação do capital humano, o que trava desenvolvimento nacional. Sem educação, não haverá capital humano qualificado como motor básico da produtividade e da competitividade.

Palavras-chave: capitalismo neoliberal; trabalho infantil; inassistência escolar; evasão escolar 